\title{
SHOULD WE CARE MORE ABOUT FRAILTY WHEN WE TREAT DIABETES?
}

\author{
L. ORLANDINI ${ }^{1}$, M. CESARI ${ }^{2}$
}

1. Azienda Servizi alla Persona Pio Albergo Trivulzio, Milano, Italy; 2. Fondazione IRCCS Ca' Granda Ospedale Maggiore Policlinico, Università degli Studi di Milano, Italy. Corresponding author: L. Orlandini, Azienda Servizi alla Persona Pio Albergo Trivulzio, Milano, Italy, laur.orlandini@gmail.com

J Frailty Aging 2020;9(2)66-67

Published online February 14, 2020, http:/ / dx.doi.org/10.14283/jfa.2020.5

Diabetes and frailty (i.e., the state of increased vulnerability to stressors) are commonly found in older individuals, often existing side-by-side. Both conditions have proven to be independently associated with negative health-related outcomes, such as hospitalization and mortality $(1,2)$. Growing interest is given to the possibility that their simultaneous presence in the individual might give life to interactions potentially affecting the clinical management $(3,4)$.

In this issue of the Journal of Frailty and Aging, FerriGuerra and colleagues (5) explore the link between frailty, diabetes and health-related outcomes. In the study, data from 763 community-dwelling Veterans affected by diabetes are retrospectively analysed. Study participants are stratified by the presence of frailty (defined according to the results of a 44-item Frailty Index (6)). In the regression model, frailty is found to be independently associated with all-cause hospitalization (Hazard Ratio [HR] 1.71; 95\% Confidence Interval [95\%CI] 1.31-2.24, $\mathrm{p}<0.001$ ) and mortality (HR 2.05; 95\%CI 1.16-3.64, $\mathrm{p}=0.01$ ), even after adjustment for potential confounders. The study suggests an important take-home message for clinicians: the identification and management of frailty may reduce healthrelated negative outcomes in diabetic patients. In other words, frailty in diabetes should be considered a special and specific target for clinical interventions.

But which are the interventions qualifying as beneficial in frail diabetic individuals? In 2017, this journal published an international position statement on the management of frailty in diabetes mellitus (7). The document provided a set of key recommendations to support clinicians in the management of diabetic patients with frailty. The panel of experts particularly stressed 1) the importance of screening for frailty in all diabetic patients, and 2) the individualisation of glycaemic targets according to the individual's frailty status. It was recommended to consider as appropriate a target range of 7.0$8.0 \%$ of $\mathrm{HbA} 1 \mathrm{c}$ in mild-to-moderate frailty, whereas a range of 7.5-8.5\% could be set as goal in case of severe frailty. The reduction of hypoglycaemia risk was also identified as the main criterion to take into account when choosing the most appropriate drug for the patient. Hypoglycaemia is associated with an increased risk of falls (8), cardiovascular events, and mortality (9). It is also subtly incumbent in frail individuals. In a recent study from MacKenzie and colleagues (10), diabetic patients with moderate-to-severe frailty had lower serum glucose concentrations compared to diabetic patients with no or mild frailty. Moreover, hypoglycaemia was observed only in diabetic patients with moderate-to-severe levels of frailty. It is thus not surprising why the position statement invited clinicians to choose metformin as first-line treatment over secretagogues drugs in particularly vulnerable patients.

Although guidelines agree that looser glycaemic control is more suitable for frail diabetic patients, the routine clinical practice still seems to miss the point. For example, in the study by Ferri-Guerra and colleagues (5), no significant difference was detected in the adherence to a tight glycaemic control between frail and non-frail patients $(52.5 \%$ vs $57.9 \%$, $\mathrm{p}=0.15)$. Frail diabetic participants were also more likely to be treated with agents exposing to a higher risk of hypoglycaemia. In fact, a significant number of frail patients with diabetes were taking sulfonylureas or insulin (57\% vs 50\%, p=0.04) compared to non-frail ones. At the same time, non-frail patients were more likely to receive metformin than their frail counterparts $(59.5 \%$ vs $41.8 \% ; \mathrm{p}<0.001)$. Consistent results were also reported by a cross-sectional study published in JAMA Internal Medicine (11), showing that community-dwelling older persons presenting both frailty and diabetes are often overtreated. In fact, it was demonstrated that nearly two-thirds of the study population was rigidly controlling glycaemia (ie, HBA1c <7\%) with the use of insulin or sulfonylureas, despite a very poor health status.

Reasons for the lack of implementation of pragmatic recommendations privileging the prevention of adverse effects over rigorous control may vary. The patient may simply not be screened for frailty. Clinicians may also find difficulties in the deprescribing process, especially when this concerns the evaluation of long-standing therapies. Guidelines developed for geriatric patients may have limited diffusion outside the geriatric field. Some drugs (i.e., sulfonylureas) may be more available and less expensive than other drugs associated with a lower risk of hypoglycaemia. Finally, it cannot be ignored that the training of future clinicians is largely based on the memorization of rigid diagnostic and therapeutic algorithms, substantially underestimating the heterogeneity of the (aging) patients.

In conclusion, diabetic patients should be routinely assessed for frailty. As Ferri-Guerra and colleagues (5) showed in their work, frailty independently contributes to the burden of diabetes in terms of healthcare utilization and decreased survival. The identification of frailty should lead to careful evaluation and potentially to an adapted intervention $(12,13)$. Clinicians should bear in mind that diabetic patients with frailty 


\section{THE JOURNAL OF FRAILTY \& AGING}

are different from the traditional diabetic patients described in the medical textbooks. They should thus be accordingly treated, always reminding the importance of "primum non nocere".

Conflict of interest: None declared by Authors.

\section{References}

1. Clegg A, Young J, Iliffe S, Rikkert M, Rockwood K. Frailty in elderly people. Lancet. 2013;381(9868):752-762. doi:10.1016/S0140-6736(12)62167-9.

2. GBD 2015 Mortality and Causes of Death Collaborators. Global, regional, and national life expectancy, all-cause mortality, and cause-specific mortality for 249 causes of death, 1980-2015: a systematic analysis for the Global Burden of Disease Study 2015. Lancet. 2016;388(10053):1459-1544. doi:10.1016/S01406736(16)31012-1.

3. Castrejón-Pérez R, Aguilar-Salinas C, Gutiérrez-Robledo L, Cesari M, Pérez-Zepeda M. Frailty, diabetes, and the convergence of chronic disease in an age-related condition: a population-based nationwide cross-sectional analysis of the Mexican nutrition and health survey. Aging Clin Exp Res. 2018;39(8):935-941. doi:10.1007/ s40520-017-0852-2.

4. Castrejón-Pérez R, Gutiérrez-Robledo L, Cesari M, Pérez-Zepeda M. Diabetes mellitus, hypertension and frailty: A population-based, cross-sectional study of Mexican older adults. Geriatr Gerontol Int. 2016;17(6):925-930. doi:10.1111/ ggi.12805.
5. Ferri-Guerra J, Aparicio-Ugarriza R, Salguero D, et al. The Association of Frailty with Hospitalizations and Mortality Among Community Dwelling Older Adults with Diabetes. J Frailty Aging. October 2019. doi:10.14283/jfa.2019.31.

6. Rockwood K, Mitnitski A. How might deficit accumulation give rise to frailty? J Frailty Aging. 2012;1(1):8-12.

7. Sinclair AJ, Abdelhafiz A, Dunning T, et al. An International Position Statement on the Management of Frailty in Diabetes Mellitus: Summary of Recommendations 2017. J Frailty Aging. 2018;7(1):10-20. doi:10.14283/jfa.2017.39.

8. Kachroo S, Kawabata H, Colilla S, et al. Association between hypoglycemia and fallrelated events in type 2 diabetes mellitus: analysis of a U.S. commercial database. J Manag Care Spec Pharm. 2015;21(3):243-253. doi:10.18553/jmcp.2015.21.3.243.

9. Amiel SA, Aschner P, Childs B, et al. Hypoglycaemia, cardiovascular disease, and mortality in diabetes: epidemiology, pathogenesis, and management. Lancet Diabetes Endocrinol. 2019;7(5):385-396. doi:10.1016/S2213-8587(18)30315-2.

10. MacKenzie HT, Tugwell B, Rockwood K, Theou O. Frailty and Diabetes in Older Hospitalized Adults: The Case for Routine Frailty Assessment. Can J Diabetes. July 2019. doi:10.1016/j.jcjd.2019.07.001.

11. Lipska K, Ross J, Miao Y, Shah N, Lee S, Steinman M. Potential overtreatment of diabetes mellitus in older adults with tight glycemic control. JAMA Intern Med. 2015;175(3):356-362. doi:10.1001/jamainternmed.2014.7345.

12. Poudel A, Hubbard R, Nissen L, Mitchell C. Are prescribing indicators essentially representing the frail older population? J Frailty Aging. 2013;2(4):217. doi:10.14283/ jfa.2013.32.

13. Rowe $\mathrm{J}$, Fried L. Incorporating frailty into clinical practice and clinical research. J Frailty Aging. 2013;2(3):126-127. doi:10.14283/jfa.2013.17 